\title{
Rapport «Traitement oral ou parentéral de la carence en fer»
}

Swiss Medical Board

Correspondance: Susanna Marti Calmell Secrétariat de l'organe responsable du Swiss Medical Board Stampfenbachstrasse 30 Case postale

CH-8090 Zurich

Tél. 0432595211

info[at]medical-board.ch
Le Swiss Medical Board a examiné la question du traitement adéquat de la carence sévère en fer. Dans son dernier rapport, le Conseil d'experts du Swiss Medical Board recommande l'administration intraveineuse de préparations de fer après une évaluation minutieuse et en traitant simultanément la cause de la carence en fer. L'organe responsable a pris connaissance du rapport et le soumet à une Post-Publication Review.

Le fer constitue un élément important du corps humain car c'est le principal constituant du pigment rouge du sang, l'hémoglobine; il est ainsi responsable de l'approvisionnement des cellules en oxygène. Le fer joue également un rôle majeur dans de nombreux processus métaboliques. Une carence importante en fer perturbe gravement le fonctionnement physique et mental (fatigue, maux de tête et troubles de la concentration) et peut provoquer une anémie. De plus, la carence en fer peut constituer un facteur de risque, notamment en cas d'intervention chirurgicale.

Bien qu'il soit en général possible de prévenir une insuffisance d'apport en fer par une alimentation riche en fer ou par des mesures permettant une meilleure absorption du fer, il faut dans certains cas compléter par un apport médicamenteux en fer. Le SMB a examiné la question de savoir si, dans de tels cas, l'administration de préparations de fer par perfusion présente des avantages pour le patient en termes d'efficacité et de qualité de vie, par rapport à un traitement oral. Ce faisant, les auteurs ont également tenu compte des possibles effets indésirables et du rapport coût-efficacité du traitement.

Sur la base de la littérature existante, le Conseil d'experts conclut que les deux modes d'administration peuvent avoir un effet positif sur les symptômes de la carence en fer. En cas de carence en fer sévère, on constate que les résultats obtenus avec le traite- ment intraveineux sont plus rapides et quantitativement plus marqués. En conséquence, le Conseil d'experts recommande:

- Chez les patients présentant une grave carence en fer symptomatique ou une anémie ferriprive, un traitement substitutif parentéral est souhaitable après un examen minutieux. Il convient, pendant l'administration du traitement, d'assurer une surveillance adéquate et d'être prêt à intervenir par rapport à d'éventuels effets secondaires.

- Il convient, dans le même temps, de mettre en place un traitement adapté de la/des cause(s) de la carence en fer.

- Il faut en outre toujours vérifier si les patients peuvent bénéficier d'un traitement parentéral de substitution en fer dans le cadre d'une intervention chirurgicale.

Informations complémentaires concernant le Swiss Medical Board en général: www.medical-board.ch, concernant le rapport du Conseil d'experts Swiss Medical Board: Rapports achevés

Post-Publication Review concernant le
rapport «Traitement oral ou parentéral de
la carence en fer»
Le rapport reflète l'appréciation du Conseil d'ex-
perts indépendant du Swiss Medical Board, basée
sur l'évidence médicale actuelle. L'organe res-
ponsable du Swiss Medical Board a pris connais-
sance du rapport et souhaite inviter les milieux
intéressés à une discussion dans le sens d'une
Post-Publication Review.
Veuillez nous adresser votre avis à l'adresse
suivante: info[at]medical-board.ch

